\title{
Deutsche Bibeln mit Druckort außerhalb des deutschen Sprachraums in der WLB
}

Deutschsprachige Bibeln werden seit dem Ersten Weltkrieg nahezu ausschließlich in den Ländern des deutschen Sprachraums gedruckt. In der Zeit davor erschienen unter historisch unterschiedlichen Rahmenbedingungen und aus vielfältigen Gründen immer wieder deutsche Bibeln im Ausland.

\section{Bibeln religiös Verfolgter}

Ein inhaltlich spezifisches Profil weisen Bibelausgaben auf, die von Deutschen gedruckt wurden, die ihre Heimat verließen, um im Ausland ihren Glauben freier bekennen und praktizieren zu können. Diese Ausgaben zielten auf Personen mit denselben oder ähnlichen Glaubensüberzeugungen ab. Sie sollten die persönliche Bibellektüre und liturgische Praxis unterstützen und dienten der inhaltlichen bzw. konfessionellen Vergewisserung.

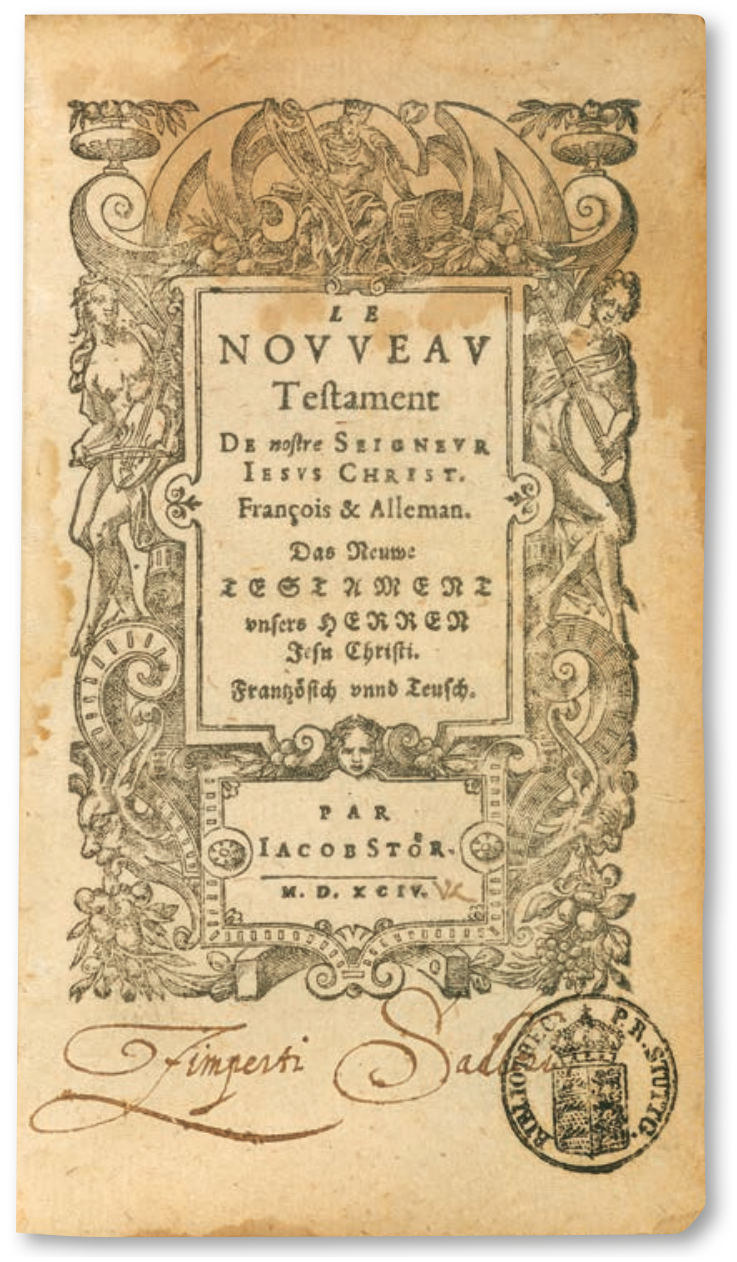

Abb. 1: Genfer Flüchtlings-Bibel (B franz. 1594 01)
Als frühes Beispiel wäre hier ein französischdeutsches Neues Testament zu nennen, das der aus Ettlingen stammende Drucker Jacob Stoer (1542-1610) 1594 in Genf herausbrachte (Abb. 1). Stoer verließ zwar schon 1559 die Markgrafschaft Baden-Baden und wurde 1568 Bürger von Genf. Seine reformierten Glaubensüberzeugungen hätten inn aber mit der seit 1569 in seiner Heimat betriebenen Gegenreformation in Konflikt gebracht. Das streng reformierte Genf war ein Zufluchtsort für französische wie deutsche Glaubensflüchtlinge, die als Adressaten einer zweisprachigen Bibelausgabe in Frage kamen. Stoer verwendete für den deutschen Teil die Luther-Übersetzung, ließ allerdings die sonst in Lutherbibeln gebräuchlichen Summarien weg, weil sie in Einzelfällen zu Kontroversen mit calvinistischer Theologie geführt hätten. In der Widmung an die aus dem reformierten Hause Oranien stammende Kurfürstin Luise Juliane von der Pfalz (1576-1644) unterstrich Stoer konsequenterweise die Bedeutung der Wahrheit der evangelischen Lehre ("la verité de la doctrine Euangelique") (S. A 2v), zu deren Erkenntnis die vorgelegte Bibelausgabe beitragen sollte.

Aus Vorsicht und zur Erweiterung des Leserkreises wurden in einer 1630 gedruckten Ausgabe des Neuen Testaments weder der Druckort Rakau (Raków) noch die Namen der Übersetzer Johannes Crellius (1590-1633) und Joachim Stegmann (15951633) genannt (B deutsch 1630 02). Im polnischen Rakau befand sich von 1602 bis 1638 die Hochschule der Anhänger des nach Polen geflüchteten italienischen Antitrinitariers Fausto Sozzini (15391604). Auch deutsche Theologen siedelten sich dort an. In der Vorrede zur Rakauer Bibeledition wird einerseits die Nähe zum griechischen Grundtext betont, andererseits die Präsentation von Übersetzungsvarianten in den Randkommentaren begründet. Darin kommt, wenn auch zurückhaltend, eine Wertschätzung der Vernunft als Urteilsinstanz für philologische, aber auch theologische Streitfragen zum Ausdruck. Der rationalistische Ansatz erschwerte den Sozinianern das Bekenntnis 
zur Dreieinigkeit Gottes. Um weniger Angriffsflächen zu bieten, wurden die Belegstellen für die Trinitätslehre in dieser Bibelausgabe (vgl. Vorrede, BI. t 8v) belassen.

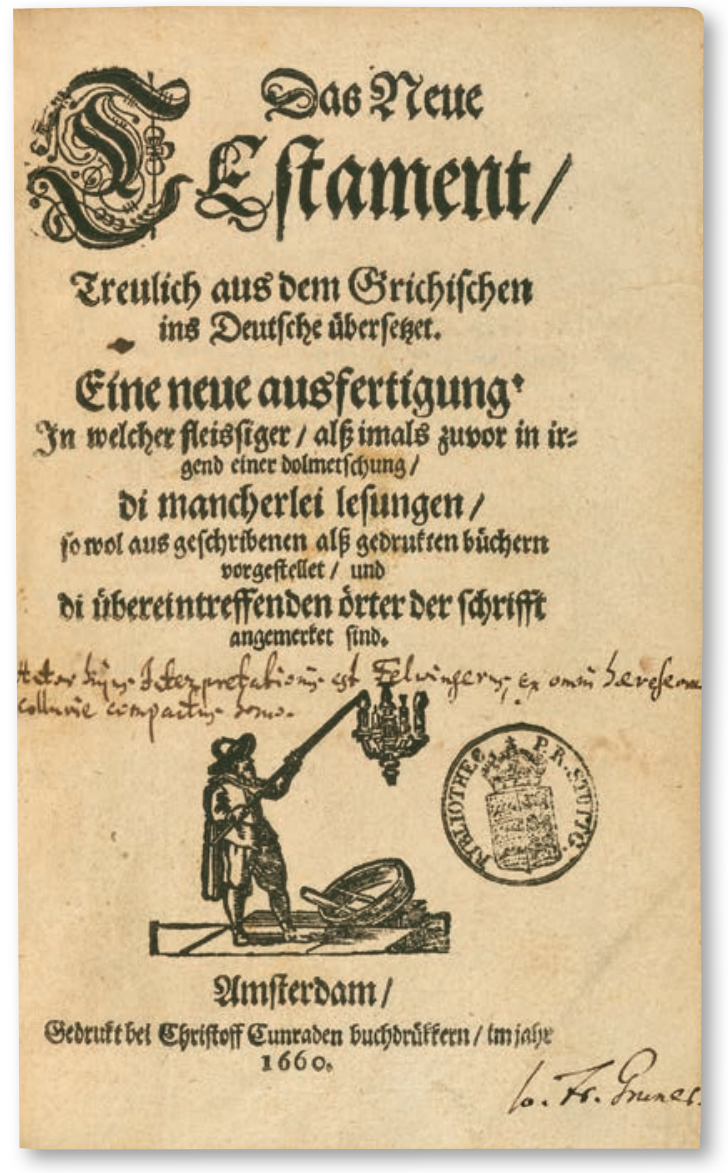

Abb. 2: Sozinianische Bibelausgabe 1660 (B deutsch 1660 01)

Wie die Rakauer Bibel wurden in einem 1660 von Christoffel Cunradus in Amsterdam gedruckten Neuen Testament alle Summarien und Einführungen in die biblischen Schriften weggelassen (Abb. 2). Gerade diese erläuternden Zusätze enthielten häufig konfessionelle Bezüge und knappe kontroverstheologische Argumente. Während der 1650er und 1660er Jahre hielt sich eine wachsende Zahl sozinianischer Exulanten in den Niederlanden, v.a. in Amsterdam, auf.' Der aus Sachsen stammende Cunradus (ca. 1615-1684) druckte Werke, deren Autoren von der jeweiligen, obrigkeitlich unterstützten Mehrheitsmeinung abwichen (z.B. Chiliasmus, Theosophie, Quäkertum). Dem entsprach seine Offenheit für eine Bibelteilausgabe, die von dem nach Amsterdam geflohenen deutschen Sozinianer Jeremias Felbinger (1616-1690) herausgegeben wurde. Felbinger nutzte für seine Neuübersetzung auch die Ausgabe von Crellius und Stegmann, setzte jedoch in lateinischer Schrift Worte hinzu, um den Text verständlicher zu machen. Für Johannes 10,30 ("Ich und der vater sind hirin eins") mag jedoch eine Abschwächung des trinitarischen Aussagegehalts intendiert gewesen sein. In der Vorrede warb Felbinger für eine Popularisierung wissenschaftlicher Werke durch deren volkssprachliche Übersetzung (S. 9) sowie für eine Gewissensfreiheit (S. 13). Indem Felbinger konfessionelle Kontroversen für "unnötig" (S. 13) erklärte, relativierte er den in zeitgenössischen Bibelausgaben profiliert vertretenen Zusammenhang von Wahrheit und Bekenntnisbindung.

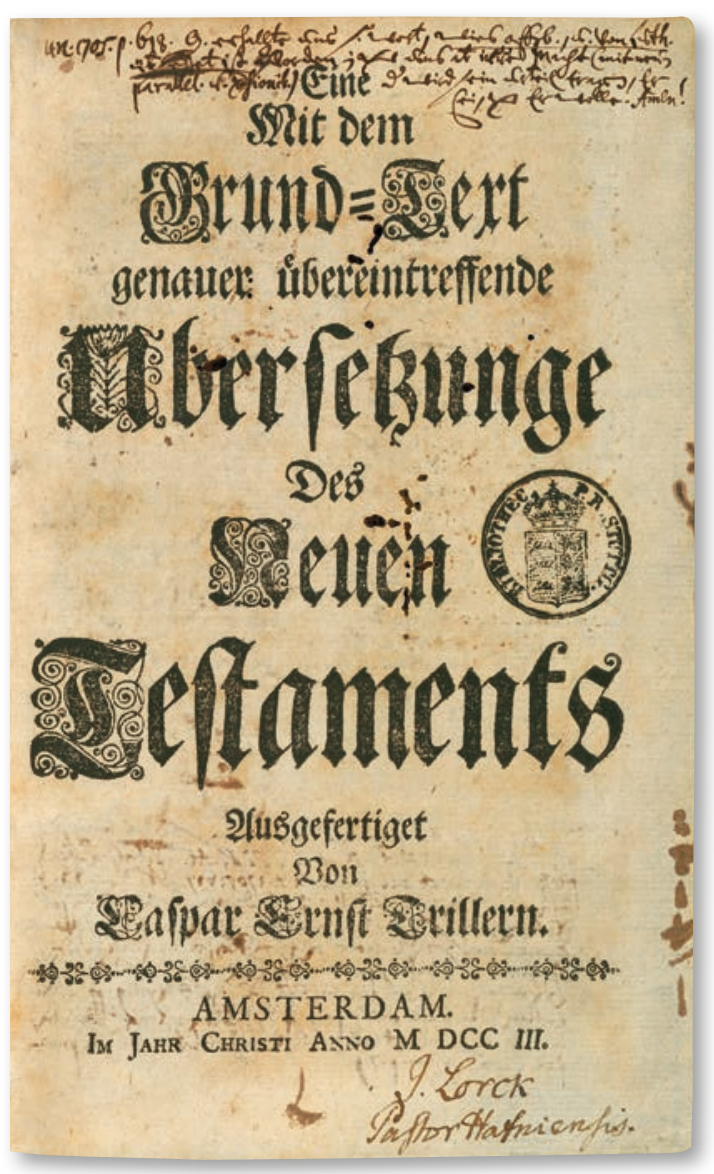

Abb. 3: Korrekturen am Luthertext (B deutsch 1703 01)

Anders verhält es sich mit Bibeln, deren Herausgeber in Deutschland bleiben, aber nicht in deutschen Orten drucken lassen konnten. So erschien 1703 „Eine mit dem Grund-Text genauer übereintreffende Übersetzunge des Neuen Testaments" in Amsterdam (Abb. 3). Der Bearbeiter Caspar Ernst Triller (1650-1717), Theologe und Philologe, bemerkte Abweichungen der Lutherbibel vom griechischen Ausgangstext. In Schwierigkeiten geriet er durch die Infragestellung einiger Übersetzungslösungen Luthers. Anders als bei den Sozinianern war inm 


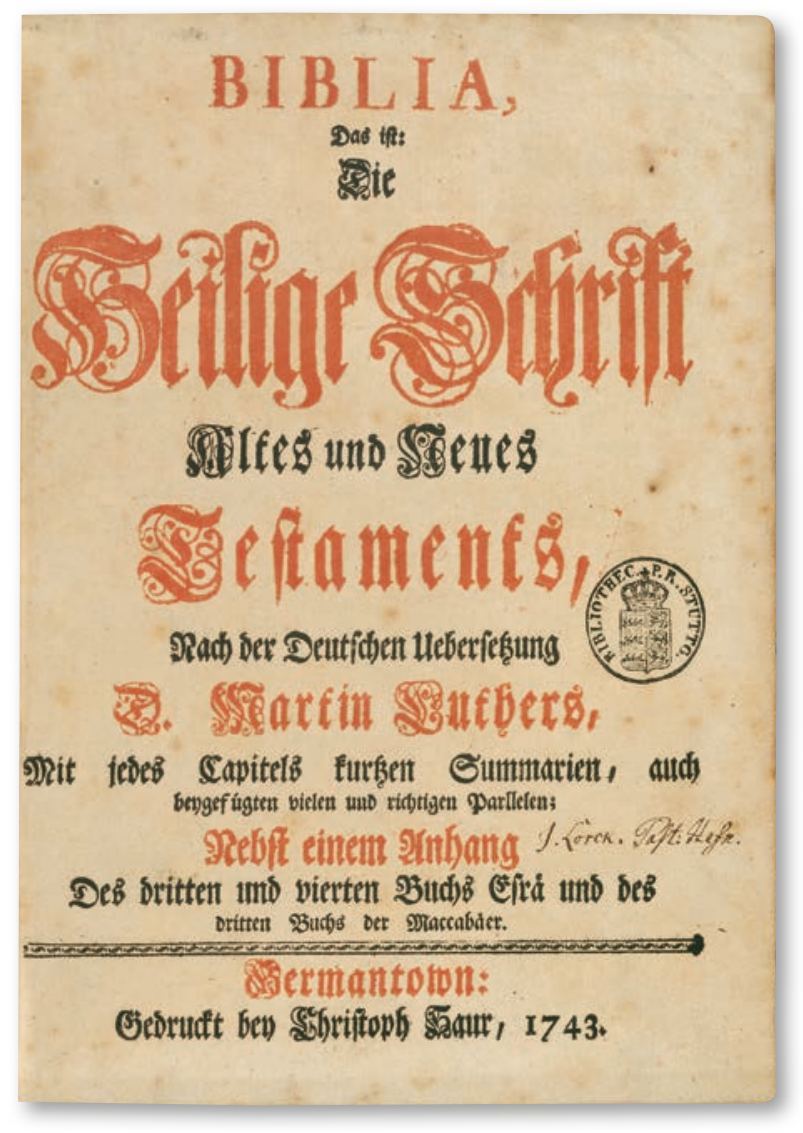

Abb. 4-5: Saur-Bibel: Titelblatt und Frontispiz (Ba deutsch 1743 01)

- wohl unter dem Einfluss des Pietismus - die philologische Exaktheit aus Gründen der Heilsrelevanz ein Anliegen. Die Stücke, "worauff der Seelen Seeligkeit beruhet" (BI. a3r), sollten "gut / rein / richtig und correct ans Licht kommen" (BI. c1r). So verzichtete er auf Summarien und bemühte sich um eine möglichst wörtliche Übersetzung.

In die entgegengesetzte Richtung lief die Motivation zu einer Neuübersetzung des Neuen Testaments, die der rationalistische Theologe Carl Friedrich Bahrdt (1740-1792) bei Johann Friedrich Hartknoch 1773 in Riga drucken ließ (B deutsch 1773 05). Ganz im Geist der Aufklärung legte Bahrdt eine sich bewusst vom Wortlaut des Grundtextes lösende Ausgabe vor und entfernte alles Anstößige. Die Bibel wollte er dadurch „beliebter machen" $(\mathrm{BI}$. )* $(6 \mathrm{~V})$, dass er zwischen Inhalt und Schale, Sachen und Worten unterschied und sich an "Gefühl und Empfindung" orientierte (BI. )*(6r). Hartknoch (1740-1789), Freimaurer wie Bahrdt, unterstützte das Anliegen durch die Typographie: Jesus-Worte sollten größer als erzählende Partien,

2) Lückel, Ulf: Johann Christoph Sauer (1695-1757), ein deutscher Drucker und Verleger in Amerika - seine ersten Jahre in Amerika und sein Netzwerk nach Deutschland. In: Soboth, Christian u.a. (Hrsg.): "Schrift soll leserlich seyn". Der Pietismus und die Medien. Beiträge zum IV. Internationalen Kongress für Pietismusforschung 2013, Halle 2016, S. 351361, hier S. 357.

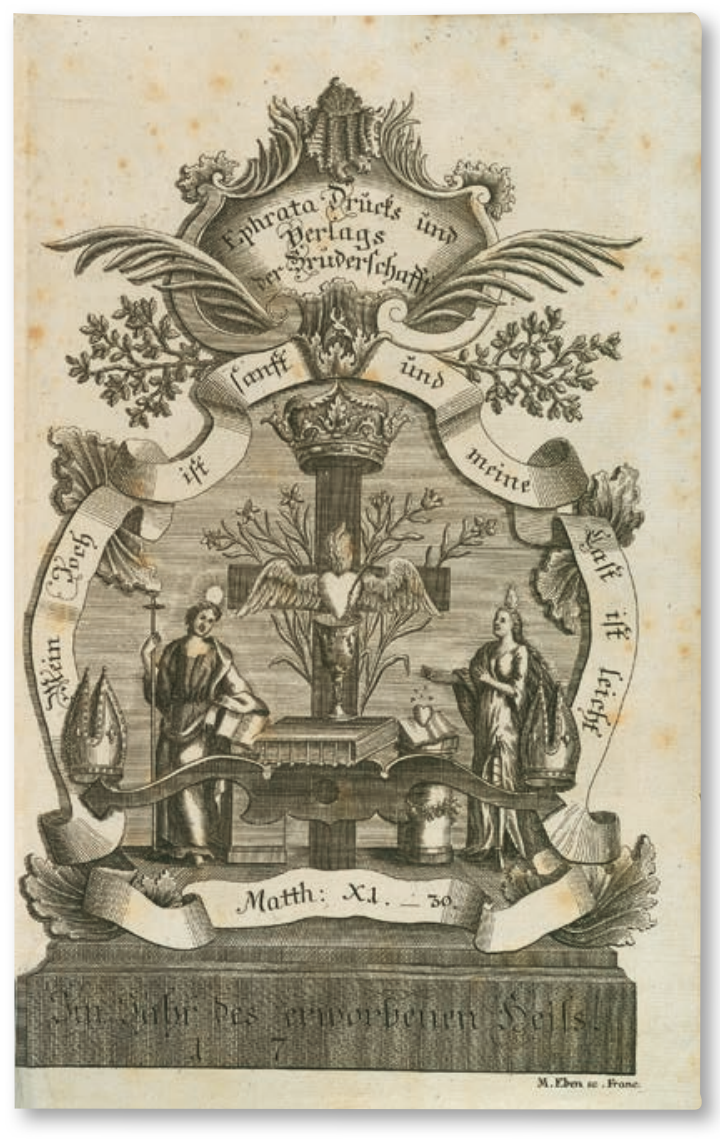

Zitate aus dem Alten Testament kleiner gedruckt werden (Bl. .*(8r). Das vorliegende Exemplar stammt aus dem Vorbesitz des Hamburger Hauptpastors Johann Dietrich Winckler (1711-1784). Die mehrfachen Amtsenthebungen Bahrdts schlossen nicht die schnelle Verbreitung seiner Werke aus.

Nicht explizit, aber faktisch war die Quart-Bibel, die Christoph Saur / Sauer (1695-1758) in Germantown (Pennsylvania) im Jahr 1743 druckte, eine Bibel für Glaubensflüchtlinge (Abb. 4-5). Der Uhrmacher und Apotheker Saur stammte aus der Kurpfalz, begab sich zunächst nach Schwarzenau (Wittgenstein), einem Zufluchtsort täuferischer Kreise. Nach seiner 1724 erfolgten Einwanderung in Amerika nutzte er weiterhin die Kontakte zu mystisch-separatistischen Strömungen des Pietismus, durch deren Vermittlung er wohl eine Druckerpresse erhielt und deren kommentierte Berleburger Bibel (1726-1742) er rezipierte. ${ }^{2}$ In der Vorrede zur Saur-Bibel, der ersten in einer europäischen Sprache in Amerika gedruckten Bibel, wurde das Problem benannt, "daß so viel arme Teutsche in diß Land kommen, welche nicht alle Biebeln mit sich bringen". Auch unter den schon länger dort Wohnenden blieben die aus Deutschland importierten Bibeln Mangelware. Dass die 34. Auflage der im Waisenhaus 
Halle gedruckten Lutherbibel möglichst genau nachgedruckt wurde, sollte als Authentizitätsbeweis gelten. Allerdings verglich Saur in seiner historischen Einführung umstrittene Bibelausgaben mit der Lutherbibel und fügte die in den Hallenser Ausgaben nicht enthaltenen apokryphen Schriften 3./4. Esra sowie 3. Makkabäer ein. Diese Schriften stammten aus der Berleburger Bibel und wurden für den Verkauf optional als Teil eines gebundenen Exemplars angeboten. ${ }^{3}$

Akzeptanz fand die Saur-Bibel unter den deutschen Einwanderern - in Pennsylvania damals über ein Drittel der Bevölkerung ${ }^{4}$ - auch wegen der identitätsstiftenden Fraktur-Schrift. Allerdings erwarben v.a. Anhänger der in Deutschland in ihrer Glaubensfreiheit bedrohten Gruppen die Saur-Bibel. Dazu 24 zählte auch die Ephrata-Kommunität, auf die ein Frontispiz des Stuttgarter Exemplars Bezug nimmt. Wegen der umstrittenen Beigaben zog sich der Verkauf der 1.200 Exemplare zwanzig Jahre bis zur zweiten, von Saurs Sohn verantworteten Auflage (1763) hin. Große Teile der 3.000 Exemplare der dritten Auflage von 1776 wurden von beiden Kriegsparteien des Amerikanischen Unabhängigkeitskrieges zum Stopfen der Gewehre verwendet, woraus sich deren Spitzname "Gun-wad Bible" erklärt. ${ }^{5}$

Aus Dank für die kostenlose Überlassung der Fraktur-Lettern durch den Frankfurter Schriftgießer Heinrich Ehrenfried Luther (1700-1770) sandte Saur zwölf Belegexemplare nach Deutschland zurück. Diese werden jedoch vor St. Malo von Piraten erbeutet und konnten teilweise aus Streubesitz von Luther zurückerworben werden. Das Stuttgarter Exemplar wurde dem russischen Gesandten Hermann Carl Graf von Keyserlingk (1695-1764) anlässlich seiner Unterkunft bei Heinrich Ehrenfried Luther während der Kaiserwahl in Frankfurt 1745 gewidmet.

Auch später bestand speziell in Pennsylvania ein großer Bedarf nach deutschsprachiger, vor allem religiöser Literatur. So druckte Johann Baer in Lancaster, Pa., ein Neues Testament (B deutsch 1821 06) sowie mennonitische Schriften. Michael Billmeyer brachte in Germantown modifizierte Nachdrucke der Saur-Bibeln heraus (z.B. B deutsch 1795 01). Auch die Swedenborgianer wären hier zu nennen, deren „Deutscher Missionsverein der Neuen Kirche in Amerika" im Jahr 1911 eine revidierte Fassung der Bibelausgabe Leonhard Tafels produzierte (B deutsch 1911 09).

\section{Bibeln für angestammte deutsche Minderheiten}

Die teilweise bzw. überwiegend deutschsprachige Bevölkerung Schleswigs und Holsteins stand seit 1460 über 400 Jahre unter dänischer Vorherrschaft. Allerdings gab es auch im dänischen Kernland und in der Hauptstadt eine deutsche Minderheit, zu der z.B. der Bibelsammler Josias Lorck (17231785) gehörte. Dies erklärt, warum in Kopenhagen deutschsprachige Bibeln erschienen, z.B. eine Psalmen-Ausgabe bei dem aus Mecklenburg eingewanderten Verleger Christian Cassube (gest. 1693) (B deutsch 1662 02).

Die meist dezidiert lutherische Prägung der Baltendeutschen ließ sich auch durch die zeitweilige Zugehörigkeit großer Teile des heutigen Lettland und Estland zu Schweden erklären. Den Baltendeutschen kam als gebildeter Oberschicht eine wichtige kulturelle Funktion zu. Eine Lutherbibel im handlichen Duodez-Format mit angehängtem Gesangbuch, die 1677 von Johann Georg Wilcke in Riga gedruckt wurde, kam auch auf Initiative des schwedischen Generalgouverneurs von Livland, Krister Horn (1622-1692), zustande (B deutsch 1677 02). In der Widmungsvorrede an das Ehepaar Horn entfaltete der livländische Generalsuperintendent Johann Fischer (1636-1705) das Bibelverständnis des erwecklichen Luthertums. Fischer veranlasste später den Druck lettischer und estnischer Übersetzungen.

Unter russischer Oberhoheit zogen besonders seit dem ausgehenden 18. Jahrhundert viele Intellektuelle aus Deutschland in die baltischen Metropolen. Dies erklärt, warum neben die traditionelllutherische Prägung zunehmend das Gedankengut der Aufklärung trat. Als Beispiel für eine v.a. an der Tugenderziehung des Menschen interessierte Bibelausgabe könnte die in Dorpat 1809 gedruckte Neuübersetzung des Neuen Testaments genannt werden, die der zuvor in Hessen tätige Dorpater Theologieprofessor Wilhelm Friedrich Hezel (17541824) erstellte (Abb. 6).

3) Vgl. Strand, Kenneth A.: Some significant Americana: the Saur German Bibles. In: Andrews University Studies 32 (1994), 1/2, S. 57-106, hier S. $68 f$.

4) Von der Lutherbibel zur Gaunergeschichte. Bücher für deutsche Einwanderer in Amerika 1728-1946, Bremerhaven: DAH, 2018, S. 4.

5) Strand, Americana, S. 71. 


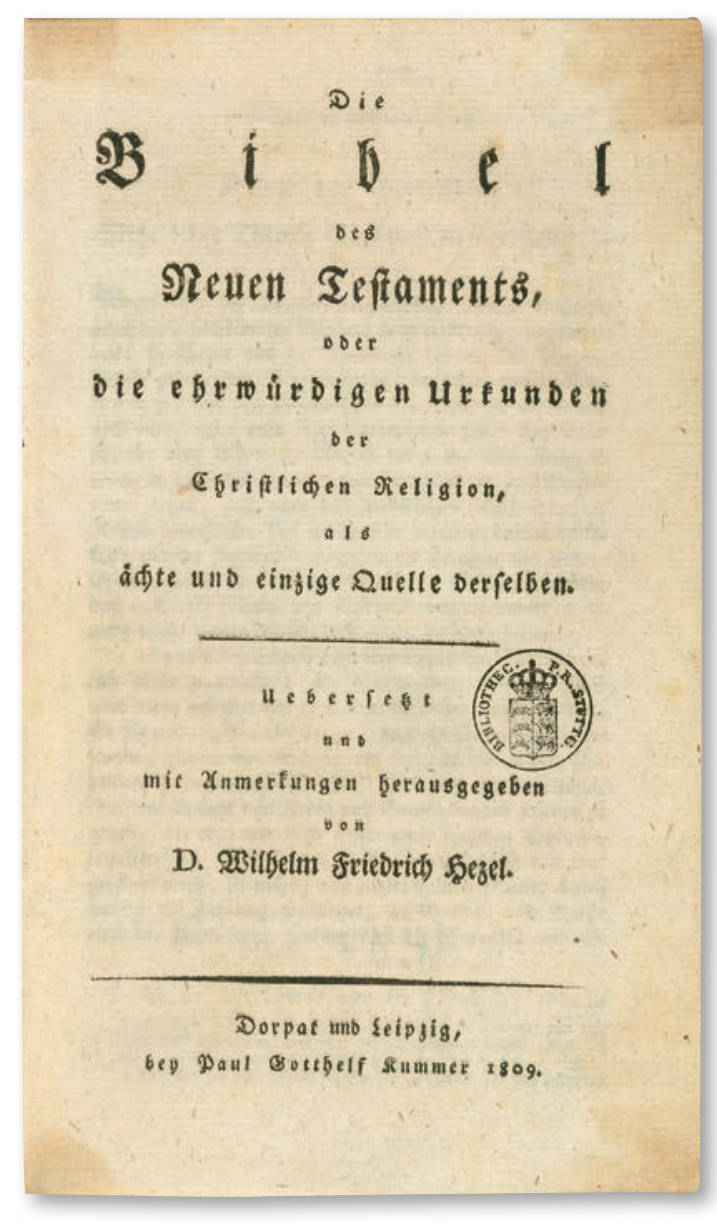

Abb. 6: Baltendeutsche Aufklärung (B deutsch 1809 01)

\section{Bibeldruck und Masseneinwanderung}

Insbesondere während des 17. Jahrhunderts, das wegen seiner wirtschaftlichen und kulturellen Blüte das Goldene Zeitalter (Gouden Eeuw) genannt wird, wanderten viele Menschen aus den Nachbarländern in die Niederlande ein. An Universitäten wie in Leiden studierten und lehrten viele Ausländer, aber auch Wirtschaft, Handel, Seefahrt, Militär wären ohne die Einwanderer nicht so erfolgreich gewesen. Leiden hatte um 1650 zu 40\% Bewohner mit nicht-niederländischem Geburtsort. ${ }^{6}$

Niederländische Drucker sahen eine kommerzielle Chance, aber auch geistliche Verpflichtung, durch Bibeldruck dafür zu sorgen, dass das „Wort Christi " „allerhand Nationen Ankömlingen und Einwohnern in ihrer eigenen Muttersprache verstendlich gepredigt und miltiglich vorgetragen werde". So formulierte der Drucker Severyn Matthysz in der Widmung seines 1646 erschienenen Neuen Testaments an den Leidener Bürgermeister seine Motivation (BI. A2v) (Abb. 7). Die große Mehrheit der

6) Vgl. Lucassen, Leo ; Lucassen, Jan: Gewinner und Verlierer: fünf Jahr-hunderte Immigration - eine nüchterne Bilanz, Niederlande-Studien 56 , Münster u.a. 2014, S. $151 f$.

7) Z.B. B deutsch 1695 01; B deutsch 1686 01; B deutsch 1696 01; $B$ deutsch 169801

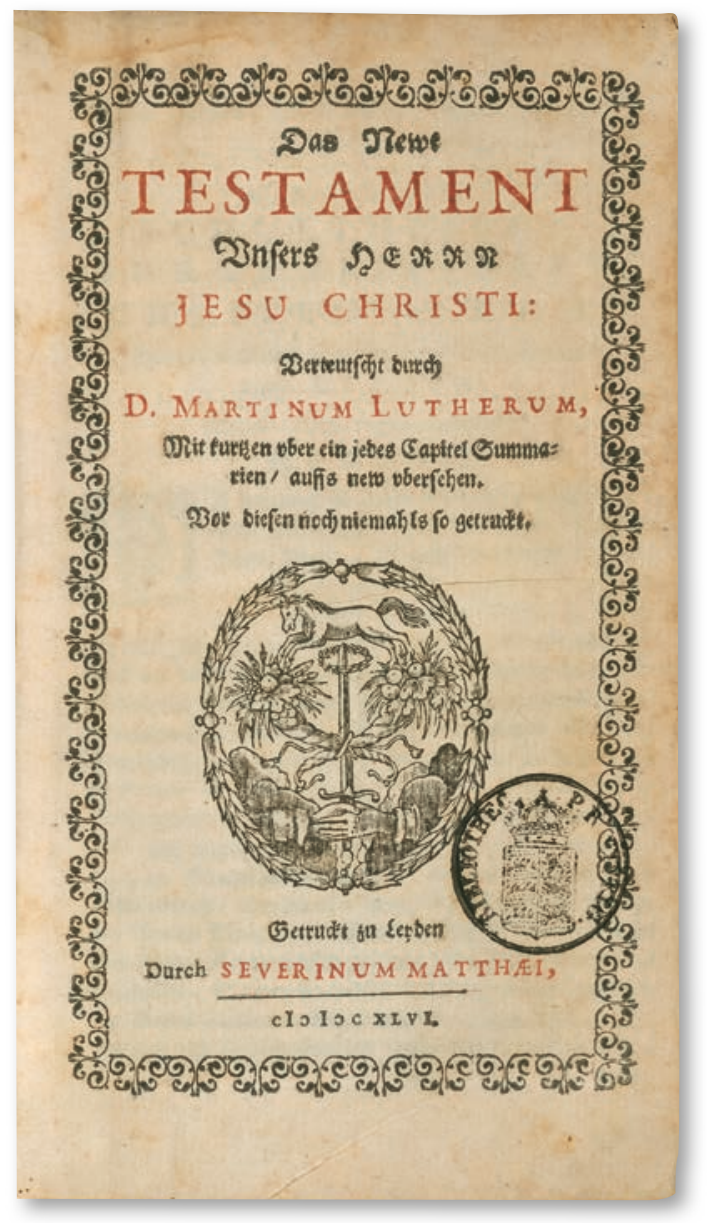

Abb. 7: Bibel für Einwanderer in den Niederlanden (B deutsch 1646 01)

Einwanderer repräsentierten die gängigen Konfessionen ihrer Heimatländer. Matthysz druckte für die Deutschen den Luthertext, allerdings in einer für die niederländischen Gegebenheiten adaptierten Form. So wurde der Luthertext angereichert mit den Erläuterungen aus der reformierten Neustadter bzw. Herborner Bibel. Der auf dem calvinistischen Genfer Psalter beruhende Psalter des Ambrosius Lobwasser (1515-1585) wurde zum Zweck des Psalmengesangs ebenso beigebunden wie der reformierte Heidelberger Katechismus.

Zurückhaltender agierte dagegen der wohl selbst aus Deutschland stammende Amsterdamer Drucker Joachim Nosche. Seine Ausgabe des Neuen Testaments von 1684 (Abb. 8) bietet lediglich den Luthertext ohne Zusätze, außerdem den Lobwasser-Psalter und ein Gesangbuch mit Luther-Liedern. Diese Ausgabe wurde wegen ihres Erfolgs mehrfach von niederländischen Druckern nachgedruckt. ${ }^{7}$

Das Nebeneinander der strukturell sehr heterogenen deutschen Bibelausgaben in den Niederlanden ist ein Indiz für die seit dem 17. Jahrhundert mit 


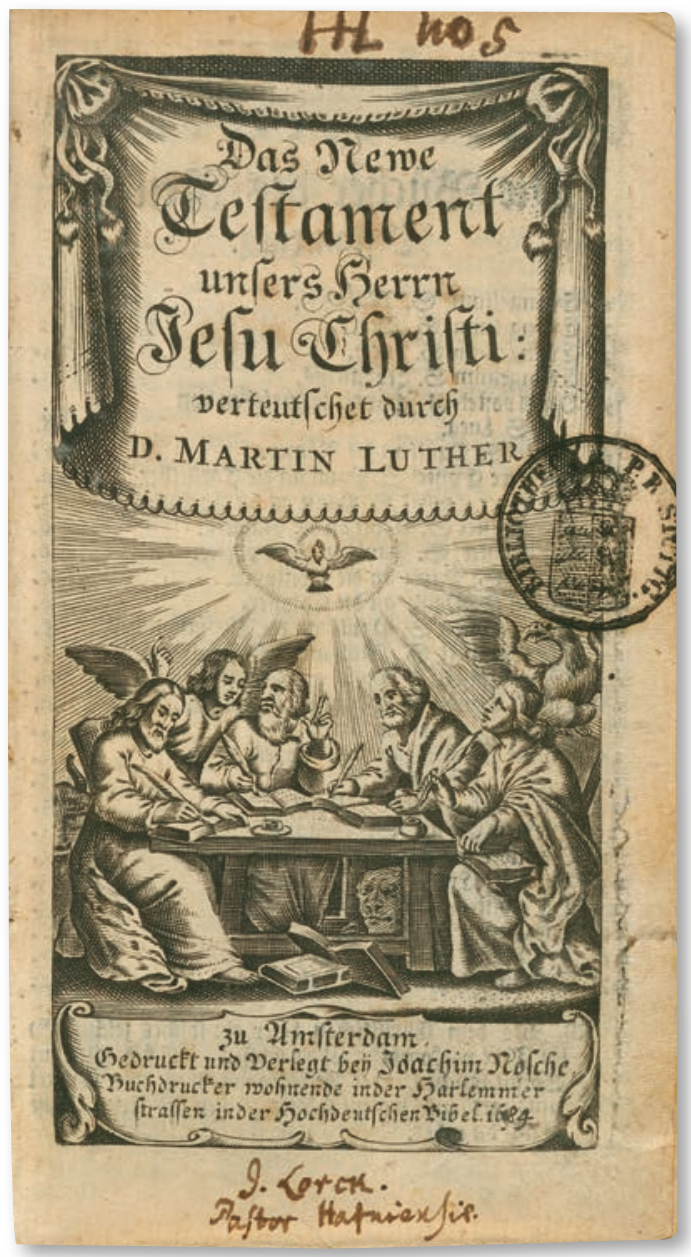

Abb. 8: Lutherische Bibel in den Niederlanden (B deutsch 1684 01)

Modifikationen begegnende spezifisch niederländische Gestalt einer pluralistischen Gesellschaft. Die starke Stellung des Calvinismus schloss die Duldung oder sogar Förderung anderer Konfessionen nicht aus. Im 19. Jahrhundert entstand das bis heute nachwirkende Säulen-System der Gesell-

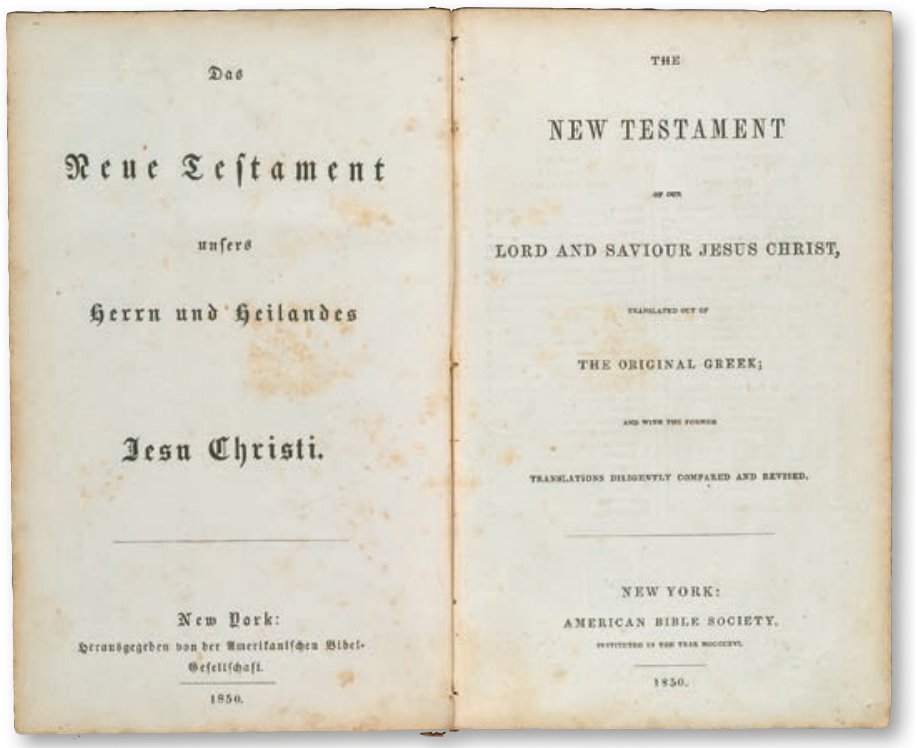

Abb. 9: Deutsch-Englische Ausgabe (B deutsch 1850 07) schaft (Verzuiling). Dieses lässt profilierte konfessionelle sowie gesellschaftliche Interessengruppen mit Parallelstrukturen in einem Nebeneinander ohne Dominanzansprüche bzw. ohne die staatliche Durchsetzung eines bestimmten Wahrheitsanspruches zu. Im Verlauf des 19. Jahrhunderts, besonders ab 1820 setzte eine Masseneinwanderung von Deutschen in Amerika ein. Auch wenn diese meist nicht wegen der größeren Glaubensfreiheit in den USA stattfand, bestand nicht zuletzt als Ausdruck kultureller Identität ein Bedarf nach christlicher Literatur als Grundlage für Gottesdienst und persönliche Frömmigkeit. Ein Anpassungsdruck, vor allem in ethnisch stärker gemischten Regionen, bestand für die Einwanderer vor allem durch die Notwendigkeit, die englische Amtssprache zu erlernen. Hilfestellung dazu sollten u.a. zweisprachige Bibelausgaben leisten, z.B. ein 1850 von der Amerikanischen Bibelgesellschaft in New York gedrucktes Neues Testament mit Luthertext und King James Version (Abb. 9).

Zwar wurden in dieser Phase auch in Philadelphia noch deutsche Bibeln gedruckt, etwa 1828 eine Lutherbibel durch Georg[e] W. Mentz (B deutsch 1828 05). Aber neben die Milieus der religiösen

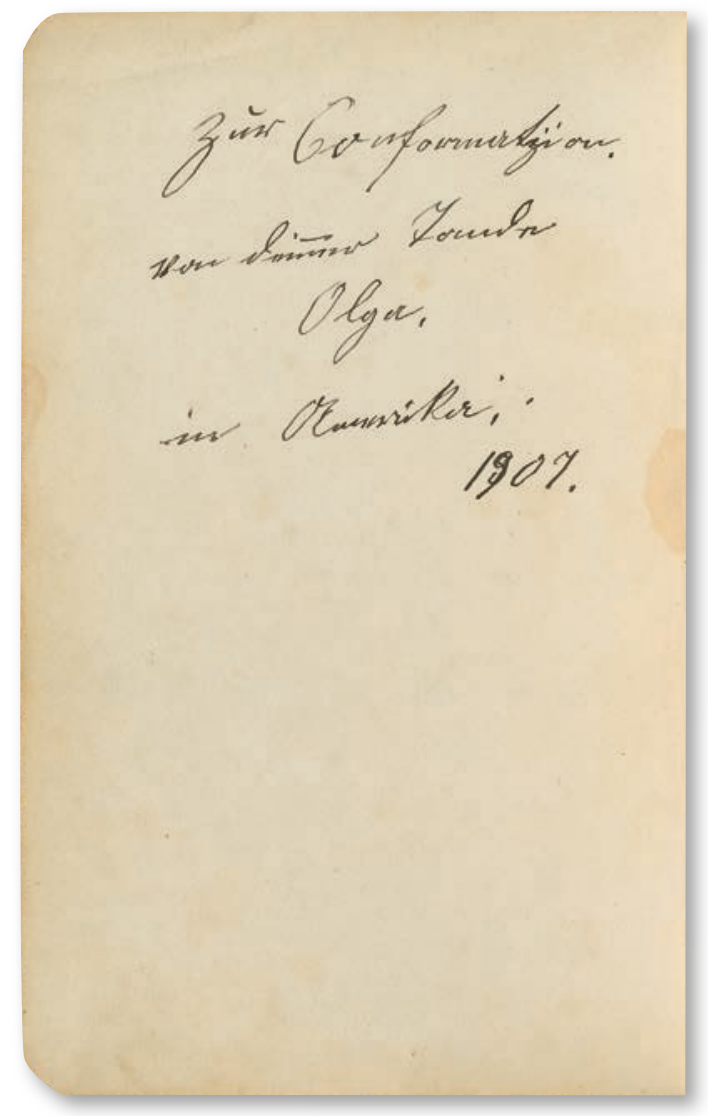

Abb. 10: Widmung in amerikanischer Bibel (B deutsch 1903 07) 
Sondergruppen in Pennsylvania traten deutsche Siedler, die sich den traditionellen Konfessionen, teilweise in deren spezifisch amerikanischer Ausdifferenzierung, zurechneten. Neue Zentren deutschen Buchdrucks standen zeichenhaft dafür. New York sollte die deutschen Einwanderer in ihrer Breite versorgen. In Milwaukee und St. Louis wurden dagegen überwiegend Bibeln für die von Deutschen geprägte, streng lutherische Wisconsin- bzw. Missouri-Synode gedruckt (z.B. B deutsch 1887 06; B deutsch 1904 10).

Die deutschen Einwanderer pflegten zumindest so lange den Kontakt zu den in Deutschland verbliebenen Verwandten, wie sie selber noch die deutsche Sprache beherrschten. So wurde z.B. eine von der Amerikanischen Bibelgesellschaft 1903 in New York gedruckte Lutherbibel mit folgender handschriftlicher Widmung nach Deutschland geschickt: "Zur Confirmation von deiner Tante Olga in Amerika. 1907" (Abb. 10).

Häufig wurden nicht nur Informationen zu Taufe, Konfirmation und Hochzeit in Bibeln festgehalten, sondern auch über Generationen hinweg Namen von Verwandten mit Lebensdaten notiert, zumal wenn diese zeitweilig die Bibel benutzten. Bemerkenswert ist, dass spätestens bei der dritten Generation der Einwanderer in den USA die Einträge vom Deutschen ins Englische wechselten. Aus Respekt für die Vorfahren wurde aber weiterhin die deutsche Bibel tradiert, bevor man in Folgegenerationen zur Benutzung englischer Bibeln überging. So wurde bei einer New Yorker Lutherbibel von 1847 (B deutsch 1847 05) der letzte, auf 1874 datierte Eintrag in englischer Sprache vorgenommen. Das Stuttgarter Exemplar der zweiten Auflage der Saur-Bibel (Ba deutsch 1763 01) weist nicht nur einen Wechsel der Sprache, sondern auch der Namensform der ersten Besitzerfamilie auf. ${ }^{8}$ Die Stiftung einer amerikanischen Lutherbibel von 1870 (B deutsch 1870 05) erfolgte nach 1893 durch einen deutschstämmigen Pfarrer, der die deutsche Sprache offensichtlich nicht mehr beherrschte. ${ }^{9}$

8) Namensform: Lefewer (deutsche Einträge: 1745 und 1767-1786); Namensform: Lefever (englische Einträge: 1794 und 1823). Bei der dritten Auflage (Ba deutsch 1776 01) finden sich für die Familie Ritter deutsche Einträge von 1779 bis 1837 sowie englische von 1833 bis 1871.

9) Der im englischen Schenkungsvermerk erwähnte Ort Ronan, Montana, heißt erst seit 1893 so: "Donated by M[i]n[ister]. Walter Morrie, Ronan, Montana, U.S.A."

10) Darlow, Thomas Herbert; Moule, Horace F.: Historical catalogue of the printed editions of the Holy Scripture in the Library of the British and Foreign Bible Society, London, 1903-1911 (DM), Nr. 4384.

11) Gelegentlich wurden alle drei deutschen Druckorte auf dem Titelblatt vermerkt (z.B. B deutsch 1888 06).
Um die deutschsprachigen Leser in Amerika leichter zu erreichen, gründeten jedoch auch deutsche Verlage Filialen vor Ort. So ließ das Bibliographische Institut Hildburghausen illustrierte Prachtbibeln auch in New York drucken (z.B. B deutsch 1830 02; Ba graph.1831 02; Ba graph.1854 01). Katholische Allioli-Bibeln des Regensburger Verlags Pustet erschienen auch in New York und Cincinnati (z.B. B deutsch 1866 08).

\section{Missionarisch motivierter deutscher Bibeldruck}

Durch Bevölkerungswachstum, Binnenmigration und zunehmende Alphabetisierung in den mitteleuropäischen Ländern konnte der Bedarf nach Bibeln mit dem traditionellen Bibeldruck nicht mehr ausreichend abgedeckt werden. Vertretern der frühen Erweckungsbewegung war die Notwendigkeit bewusst, breiten Bevölkerungsgruppen den Zugang zum Wort Gottes durch persönliche Bibellektüre mit erschwinglichen Bibelausgaben zu ermöglichen. Der Aufbau der kontinentalen Bibelgesellschaften ging auf die Initiative der 1804 gegründeten British and Foreign Bible Society (BFBS) zurück.

Zunächst gab die BFBS in London den Druck deutscher Bibeln bei dem eingewanderten deutschen Drucker Johann Benjamin Gottlieb Vogel (gest. 1832) in Auftrag. So erfolgte der Nachdruck der Hallischen Ausgabe von 1805 in London „auf Kosten der Großbritannischen und Ausländischen Bibel-Gesellschaft" (Abb. 11). Später ging der von der BFBS in London finanzierte deutsche Bibeldruck auf den einheimischen Drucker William Mavor Watts (17971874) über. Die württembergische Provenienz einer Taschenausgabe von 1846 (B deutsch 1846 01) belegt die Effizienz der Verbreitung der Londoner Ausgaben in Deutschland.

In einer Übergangsphase finanzierte die BFBS Bibeldruck und -verbreitung durch deutsche Verlage. So wird zu einer 1855 in Sulzbach gedruckten Ausgabe des katholischen Übersetzers Leander van Eß (1772-1847) in den Akten vermerkt: "Circulated in this form by the B. F. B. S. ") ${ }^{10}$ (B deutsch 1855 02).

In der zweiten Hälfte des 19. Jahrhunderts errichtete die BFBS Depots bzw. Filialen in Frankfurt/Main, Köln, Berlin und Wien. ${ }^{11}$ Bei Material, Transport und Lagerung ließ sich der Bibeldruck dadurch effizi- 


\section{Das}

\section{Peue Tegtament} unjers

55ertm unto 5eitanbes

Iefu anलs 3 की $\underline{\underline{12}}$

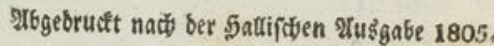

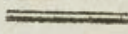

Abb. 11: Deutsche Bibel aus London (B deutsch 1811 01)

enter gestalten. Es erschienen Voll- und Teilbibelausgaben in unterschiedlichen Formaten, meist Lutherbibeln, aber auch katholische Ausgaben. Neben ärmeren Personen zielte man auf die Verteilung von Bibeln in Krankenhäusern, unter Soldaten und Strafgefangenen. ${ }^{12}$ Analog zur BFBS gründeten auch die Amerikanische bzw. die Schottische Bibelgesellschaft Zweigstellen in Hamburg bzw. Bremen (z.B. B deutsch 1896 02; B deutsch 1880 03). Noch nicht mit Beginn des Ersten Weltkriegs, aber in dessen Verlauf kam der deutsche Bibeldruck im Ausland bzw. im Auftrag ausländischer Institutionen zum Erliegen. Deutsche Kultur und Sprache wurde im angloamerikanischen Raum mit dem Feind assoziiert. Immerhin wurde noch 1916 eine BFBS-Bibel in Berlin gedruckt (B deutsch 1916 05).

Auf neutralem Boden wurden Bibeln für die Seelsorge unter deutschen Kriegsgefangenen des Ersten Weltkriegs gedruckt. So erschien 1915 im Auftrag des CVJM-Weltkomitees in Genf eine

\section{Jesus don Mazareth}

\author{
(5vantgelientharmontic \\ nach ben vier Evangelien, nit 4 Starten unb cinem Blan \\ vont \\ 9lerander gBeitphal, Dr. theol. \\ Broi. bon. ber ltniverität von Ioulouic
}

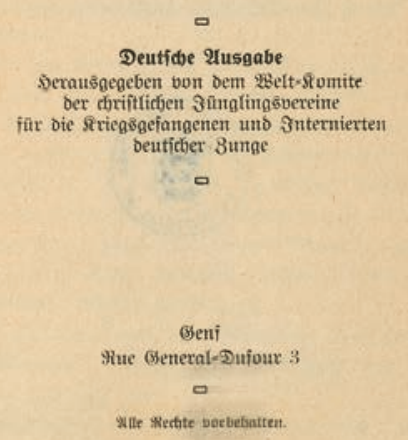

Abb. 12: Kriegsgefangenenbibel (B deutsch 1915 29)

Evangelienharmonie "für die Kriegsgefangenen und Internierten deutscher Zunge" (Abb. 12). Es handelte sich um die deutsche Übersetzung eines Werks des französischen Theologen Alexandre Westphal (1861-1951). Nach Rückkehr eines Kriegsgefangenen gelangte das Stuttgarter Exemplar zunächst in den Besitz des CVJM Bruchsal. ${ }^{13}$

Wegen der sprachlichen Verwandtschaft sind hier auch die jiddischen Übersetzungen des Neuen Testaments zu nennen, die vor allem in den 1820er Jahren im Auftrag der anglikanischen London Society for Promoting Christianity among the Jews (auch: London Jews Society) gedruckt wurden. Die Ausgabe von 1820 (B jidd.1820 01) basierte auf einer Frankfurter Lutherbibel von 1819 (B deutsch 1819 02), bezog sich in der Titelformulierung auf den "Messias" und sollte das Christuszeugnis unter Juden unterstützen.

Christian Herrmann

12) Familieneinträge z.B. in: B deutsch 1878 01; B deutsch 1884 07. Mit Stempel einer Strafanstalt: z.B. B deutsch 1873 02; B deutsch 190611 Mit Exlibris eines Soldaten: z.B. B deutsch 1891 03. Vgl. Canton, William: The story of the Bible Society, London 1904, S. 132.

13) Der Stempel eines französischen Kriegsgefangenenlagers für Offiziere in B deutsch 185103 weist darauf hin, dass auch ältere, in Deutschland gedruckte Taschenbibeln gelegentlich von Soldaten mitgeführt wurden. 